\title{
Bot Detection in Reddit Political Discussion
}

\author{
Sofia Hurtado \\ Carnegie Mellon University \\ Pittsburgh, Pennsylvania \\ shurtado@andrew.cmu.edu
}

\author{
Poushali Ray \\ Carnegie Mellon University \\ Pittsburgh, Pennsylvania \\ poushalr@andrew.cmu.edu
}

\author{
Radu Marculescu \\ Carnegie Mellon University \\ Pittsburgh, Pennsylvania \\ radum@cmu.edu
}

\begin{abstract}
The existence of social media bots on political forums can muddle the perception of public opinion. Bot detection has been successful on platforms such as Twitter, Facebook and Youtube. However, our research focuses on characterizing suspicious behavior on the social media platform Reddit; this platform structurally differs from its peers in that users subscribe to page topics and contribute to the discussion through comments or topical posts. We hypothesize that persons who intend to influence the public opinion would deploy paid users, or social media bots, to artificially amplify a political sentiment. We validate our hypothesis by using a networkbased approach that reveals a fully connected band of users which includes users with the word "bot" in their name, as well as users who exhibit otherwise a bot type behavior.
\end{abstract}

\section{CCS CONCEPTS}

- Networks $\rightarrow$ Network types; Social media networks; Overlay and other logical network structures;

\section{KEYWORDS}

Social Media Networks, Bot Detection, Reddit

\section{ACM Reference Format:}

Sofia Hurtado, Poushali Ray, and Radu Marculescu. 2019. Bot Detection in Reddit Political Discussion. In Proceedings of Fourth International Workshop on Social Sensing (SocialSense'19). ACM, New York, NY, USA, 6 pages. https://doi.org/10.1145/3313294.3313386

\section{INTRODUCTION}

Social media bots and paid human agents were deployed in an effort to influence the United States 2016 presidential election [12]. Most research focuses on detecting bots on Twitter and Facebook, however paid human agents are often undetected. In this paper, we develop a network-based approach that focuses on user comments in order to identify suspicious users on an under-researched platform, namely Reddit.

The Reddit platform is structured to have "subreddits", which are content feeds focused on a topic where members can post related content. Each subreddit has its own "Auto Moderator" which is a social media bot that automatically regulates the posted content and user's comment language. Users are free to post onto a

Permission to make digital or hard copies of all or part of this work for personal or classroom use is granted without fee provided that copies are not made or distributed for profit or commercial advantage and that copies bear this notice and the full citation on the first page. Copyrights for components of this work owned by others than ACM must be honored. Abstracting with credit is permitted. To copy otherwise, or republish, to post on servers or to redistribute to lists, requires prior specific permission and/or a fee. Request permissions from permissions@acm.org.

SocialSense'19, April 15, 2019, Montreal, QC, Canada

(C) 2019 Association for Computing Machinery.

ACM ISBN 978-1-4503-6706-6/19/04 . \$15.00

https://doi.org/10.1145/3313294.3313386 subreddit feed, as well as comment on other posts in that feed. Users are also permitted to deploy social media bots onto friendly "subreddits" and are encouraged to follow the crowdsourced etiquette for Reddit bots known as "Bottiquette" [4]. Notably, developers are encouraged, but not enforced, to include the word "bot" in their bot account username. As a test case, we target the bot identification of subreddit "The_Donald" which became the known subreddit hub for Donald Trump supporters during his 2016 campaign.

\section{PREVIOUS WORK}

Methods used for bot detection in the existing literature include network based, crowdsourcing, and feature based approaches [8]. One such graph-based approach is deployed in SybilRank which analyzes the likelihood that a Facebook account is fake [3]. Alternatively, The BotOrNot service, designed for bot detection on Twitter, uses machine learning techniques to judge whether an account is controlled by a bot based on six feature dimensions: network, user, friends, time, content, and sentiment [5]. Combination of multiple features enables efficient bot detection.

Most work on bot detection deals with machine learning techniques on platforms like Twitter and Facebook. Deep neural networks [2], Bayesian regularized neural networks [10], fuzzy logic [7], and DNS flagger [1] are some other approaches which have been tested. They use metrics like abnormal account activity, ratio of engagement (high upvote/downvote activity, less actual comment, large following with minimal engagement), percentage of followers with newly created accounts as well as ratio of URLs in posts [6].

Research pertaining to Reddit bots focuses on characterizing their diverse taxonomy and functionality in adding to Reddit's playful bot culture [11]. Furthermore, researchers aiming to detect Reddit bots utilize temporal analysis to detect accounts that do not behave in the Rest-Sleep-and Comment pattern exhibited by humans [9].

We note that both Twitter and Facebook are platforms that promote user-to-user subscription, unlike Reddit which is based on user-to-topic subscription. We extend temporal analysis bot detection by exploiting this structural platform difference which offers a new element to graph-based bot detection.

\section{APPROACH}

We propose a new network-based approach to identify suspicious behavior on an understudied social media platform: Reddit. The Reddit platform offers a new structure to explore online social communities. Because the Reddit website is a tree of topics that users subscribe to, the community engages in discussion surrounding that topic. Subreddits are the topical pages, or feeds, and posts are 
the content posted to the pages. Users can comment on posts, as well as submit their own posts to the subreddit pages.

The dataset we consider consists of all of the comments made by users on every subreddit within Reddit binned for one month. We focus our attention on political discussion surrounding the 2016 election. As a case study, we chose the subreddit page "The_Donald" to focus on those Reddit commentators who supported Donald Trump's candidacy. Because of the Russian influence campaign, more suspicious behavior is likely to exist in this Donald Trump online community and this online entity can serve as a good, contained baseline to characterize bots and paid human agents.

Our research interest lies within investigating whether scrutinizing the network behavior of the online sub-communities can reveal distinguishable groups of suspicious users. Our intuition leads us to believe that disingenuous users, either bots or paid human agents, are a subset of suspicious users who work in packs to actively spread media content. Likely those banded fake accounts comment on the same posts in order to promote the content's popularity and influence popular opinion. For example, if there are 10 posts with 100 users commenting on them and 6 of the users commented on all 10 posts together, we hypothesize that these are suspicious users.

In order to find the highly connected users, we create two networks. The first network consists of posts that are connected to other posts if they share the same users; in this model, the edge weights represent the number of shared users. The second network consists of users that are connected if they commented on more than one of the same post; in this second model, the edge weights represent the number of comments in shared posts.

The first model's post-to-post network acts as the functional net- work that simplifies the dataset of users. Rather than creating a fully connected user graph for each post, we only care about users who have commented on more than one post together. Therefore, the first pass through the data identifies the sets of posts that share users. Then, we connect users found in those shared lists to form the second model. The user-to-user network model reveals the structural connectivity between users.

We then analyze the users within the user-to-user network with the highest edge weights for their down time between comments. We hypothesize that users with the highest edge weights will have smaller down time between comments than the average user which would indicate that the user in question is likely a bot.

\section{EXPERIMENTAL SET UP AND RESULTS}

\subsection{Network Analysis}

4.1.1 Post-to-Post Network Model. Figure 1 represents the post network where posts are connected if they have shared users.There are 407 total number of posts found within 2,000 comments; of those, 303 posts share at least one or more users. The maximum number of shared users between two posts is 6 users.

Intuitively, it could be that a large portion of the posts share users because one user might be vocal on multiple posts. The inflated edge weights on the post-to-post network model therefore contextualize the overall popularity of the post rather than malicious behavior. For example, it might be the case that two very popular posts received a lot of comment traffic and share a lot

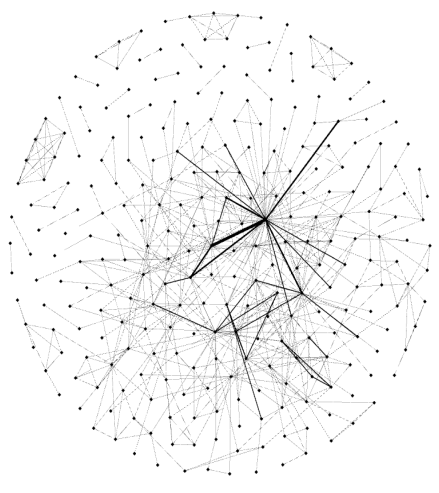

Figure 1: Post-to-Post Network resulting from 2,000 comments originating from "The_Donald” i n November 2016. This weighted graph (i.e.,dark lines have higher weights) consists of nodes that are posts. Edges are created when one user comments on at least two posts and increment on each occurrence.The large connectivity of some nodes indicates that at least one user is commenting on many posts.

of users. Their high number of shared users will result in a high edge weight in the post-to-post network. Yet, perhaps only two of those shared users are found commenting multitudes of the same posts. Therefore, the post-to-post network is not useful in identifying the hyper-connected users however it does serve a purpose as a user- to-user network construction tool. Subsequently, looking closer into the actual users who are shared among posts reveals interesting results.

4.1.2 User-to-User Network Model. Two users are connected if they commented on more than one of the same posts. As seen in Figure 2, the total number of users found in 2,000 comments is 1110 , with only 80 of them being connected. The thickness of the edges are drawn in accordance to their edge weight. In Figure 2, all edges have the value of 1 with the exception of the thickest edge which has the value of 3 . This indicates the maximum number of posts two users commented on together is 3 posts between user "IncarceratedMascot" and "FlareKR". The username of the most highly connected user is "TrumpTrain-bot". Though this user does not have the heaviest edge weight to another user, it does have the most connections (11). However, when expanding the comment dataset to parse 200,000 comments, the user network indicates that the user "TrumpTrain-bot" has the highest edge weight amongst all users.

We apply the same method to a larger dataset of 200,000 comments and expand the timescale to include previous months leading up to the 2016 election and those that followed. We choose to perform this network analysis over 15 months with an interval of 3 months that follow the crucial moments in the election timeline.

4.1.3 August 2016. Figure 3 shows the User-to-User network for August 2016, subreddit "The_Donald", with the most highly connected nodes and their respective edges colored in red. The 


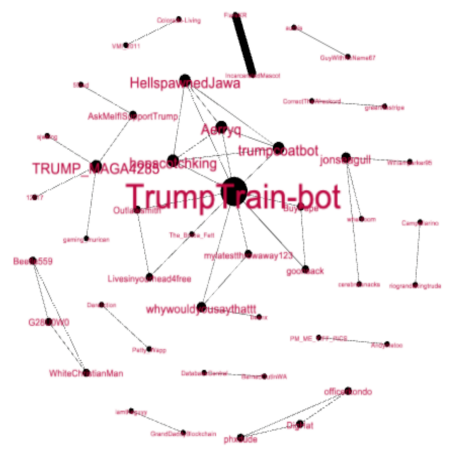

Figure 2: User-to-User Network resulting from 2,000 comments originating from subreddit "The_Donald" in November 2016. The nodes are users and the edges are the number of identical posts they jointly commented on; their edge weights increase on each occurrence. The node label sizes are correlated to their weighted degree. The user "TrumpTrain-bot" has the largest weighted degree of 11.

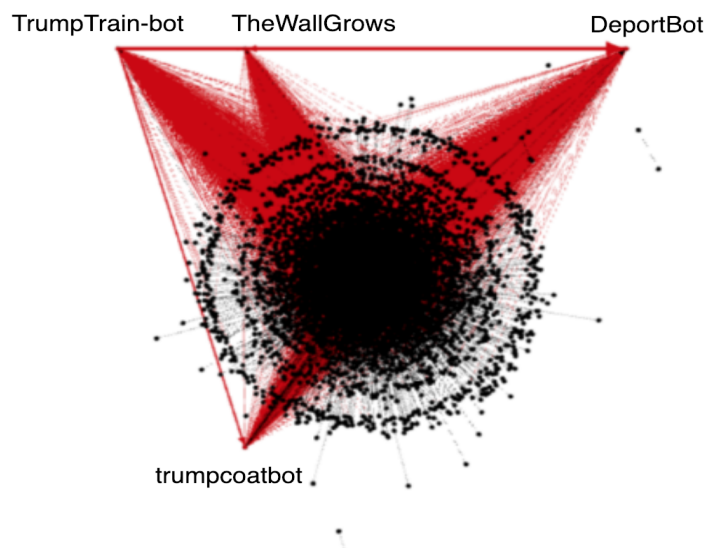

Figure 3: User-to-User network from 200,000 comments in “The_Donald”, August 2016 (nodes=9158,edges=664340, clustering coefficient $=0.355$, average degree: 75.54 , average path length:2.54).

user "TrumpTrain-bot" shares the highest edge weights with "DeportBot" (8128), followed by edge weights of 3741 and 2850 connecting "TrumpTrain-bot" to both "TheWallGrows" and "trumpcoatbot" respectively. "TrumpTrain-bot" and "DeportBot" are the top two users with the highest node degree; notably, "DeportBot" ranked 15th in betweenness centrality with a value of 221542.82 . These network parameters prove helpful in quantifying the suspicious users within connectivity of the network. For instance, the users with highest ranking edge weights that contain the word "bot" in their usernames as directed by Reddit's Bottiquette, also bring to the forefront highly connected users without the word "bot" in their name such as "TheWallGrows".

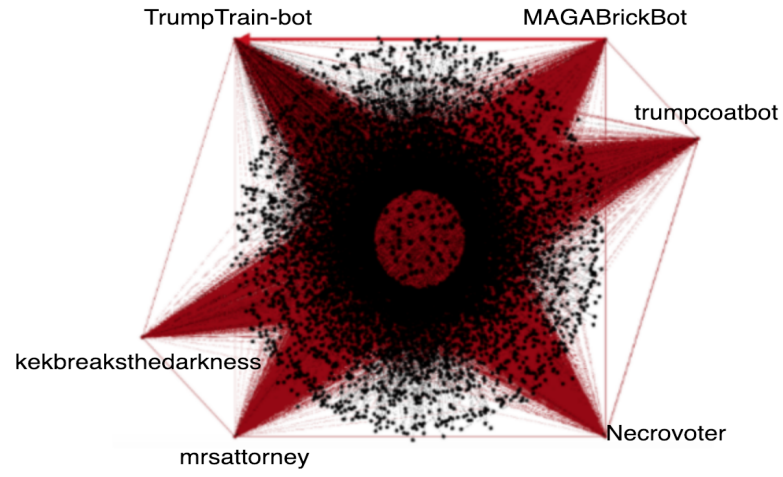

Figure 4: User-to-User network from 200,000 comments from “The_Donald", February 2018 (nodes $=10544$,edges $=858961$, clustering coefficient:0.319, average degree: 81.464 , average path length:2.548).

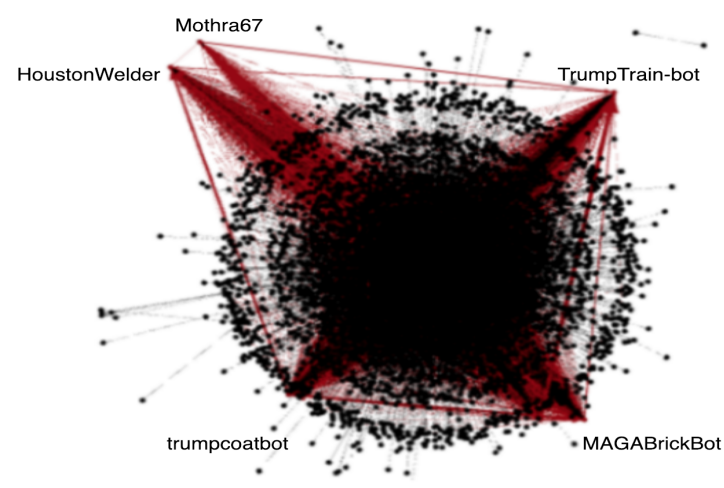

Figure 5: User-to-User network from 200,000 comments from "The_Donald", May 2018 (nodes=10623, edges=565549, clustering coefficient:0.311, average degree: 53.238 , average path length: 2.607$)$.

4.1.4 February 2018. In Figure 4, the top three highest edge weights are between "MAGABrickBot", and "TrumpTrain-bot", "trumpcoatbot" and "Necrovoter", respectively. The values are 1711, 1035 and 496 for the three edge weights, respectively. "MAGABrickBot" has the highest degree (value 4744), whereas "TrumpTrainbot” is the top user for betweenness centrality (value 2066378.16).

4.1.5 May 2018. Figure 5 shows the results for May 2018. The highest edge weight is between "MAGABrickBot" and "TrumpTrainbot" with a value of 1225 . The second highest edge weight is 861 between "MAGABrickBot" and "trumpcoatbot". Lastly, the third highest edge weight is 820 between "HoustonWelder" and "Mothra67 ". Notably, "TrumpTrainBot" ranks fourth in the overall order of users with highest degree and "Mothra67" has the highest betweenness centrality index amongst all users.

The resulting networks from August 2016 (Figure 3), February 2018 (Figure 4), and May 2018 (Figure 5), unearth the users with the largest weighted connections. Of the top three highest edge 


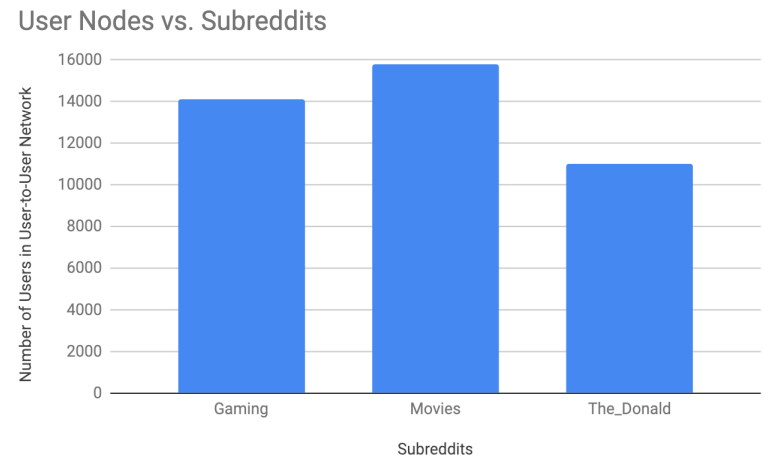

Figure 6: Number of user nodes within the User-to-User network acquired from 200,000 comments for each subreddit page: “Gaming", "movies", and "The_Donald" in November 2016.

weights, each network linked accounts that identify as bots to accounts that did not include "bot" in their name. These such users are deemed suspicious because they share behavioral attributes with known "bots", yet they are not explicitly identifying as bots. Whether the account is determined to be adversarial depends on the effect their commenting on targeted posts affects the communal discussion as a whole.

In a user-to-user social network subscription model, such as Twitter, an adversarial user aiming to target another user can utilize point to point communication by "friending" them or baiting them to return a "follow". However, in the user-to-topic subscription model found in Reddit, the adversarial user must repeatedly infect the shared content feed in hopes of inflating the popularity of the post which in turn increases the likelihood that the target user will see the promoted content. This could explain the high connectivity between accounts containing the word "bot" in their usernames.

4.1.6 Edge Weight Analysis. After noticing that the top two users in each month's user network has the word "bot" embedded in their username, we wonder if this would hold true for other subreddit pages. Furthermore, we want to give the maximum edge weight context by comparing the value with the maximum edge weight from other comparable subreddits.

To investigate whether other subreddits would reveal the top ranking users to have the word "bot" in their usernames, we then look at other popular subreddits during the same time frame, namely November 2016, in order to judge whether "The_Donald" network is commonplace or notable. The other two subreddits that have available the same amount of comments available are "Gaming" and "Movies". As seen in Figure 6, "Gaming" results in 14,113 connected users, "Movies" results in 15,802 connected users, and "The_Donald" results in 11,037 connected users. Figure 7 visualizes the maximum edge weight between any two users in their respective subreddit networks. "Gaming" has a maximum edge weight of 136; "Movies" has a maximum edge weight of 190; "The_Donald" has maximum edge weight of 3,240. Notably, the amount of connected users in both "Gaming" and "Movies" subreddit outnumber the

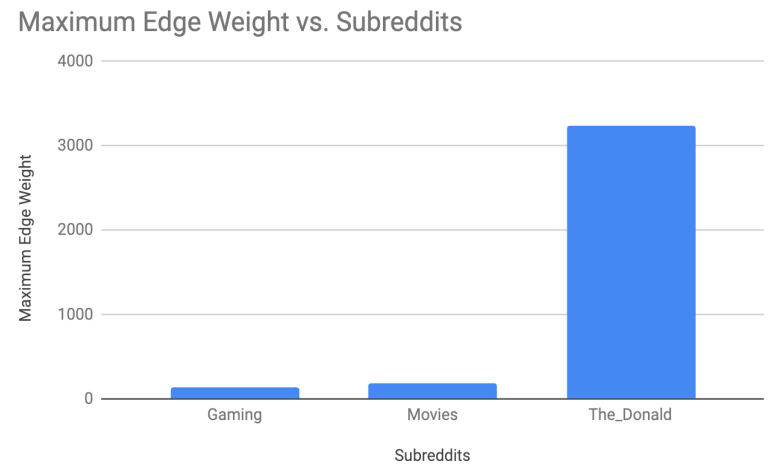

Figure 7: The maximum edge weight between any two users within the User-to-User network acquired from 200,000 comments for each subreddit page: "Gaming", "movies", and “The_Donald" in November 2016.

users in "The_Donald", yet "The_Donald" produces a very large maximum edge weight and has 96 other edge weights above 190 . This makes us conclude that the maximum edge weight is an effective way to detect abnormal behavior within the Reddit platform which may indicate the infiltration of bots and adversarial users in a subreddit. Though it can be argued that a political discussion can evoke more involvement between two users, say during a heated discussion, the average time between comments (when applied to the users with the highest edge weights) suggests that the users are not human but rather bots. A future task would be to broaden this analysis across the political spectrum and compare politically charged subreddits with the same amount of users within 200,000 comments.

Maximum edge weight between all connected users in each month is also plotted in Figure 8. Two spikes can be observed namely, one in February 2016 and the other in August 2016. The spike in the February 2016 can possibly be explained by Donald Trump winning the Iowa Caucus on February 1st. The spike in August 2016 can also possibly be explained by Donald Trump becoming the Republican presidential nominee between the months of May and August. Tracing the values over two years down the 2016 elections shows that the maximum edge weight gradually reduces to pre-election levels. Thus, the connectivity of the networks seems to spike during the election period of 2016 .

\subsection{Temporal Analysis}

After concluding that the maximum edge weight metric is unique to "The_Donald", we investigate whether we can tie other known elements of bot behavior to the maximum edge weight value. The users found to be with the higher maximum edge weights are then processed to calculate their average time between comments through their entire Reddit social media presence. This allows us to see how often within a month they post comments, as well as how many other subreddits they are active in and whether those other subreddits are political or apolitical. 


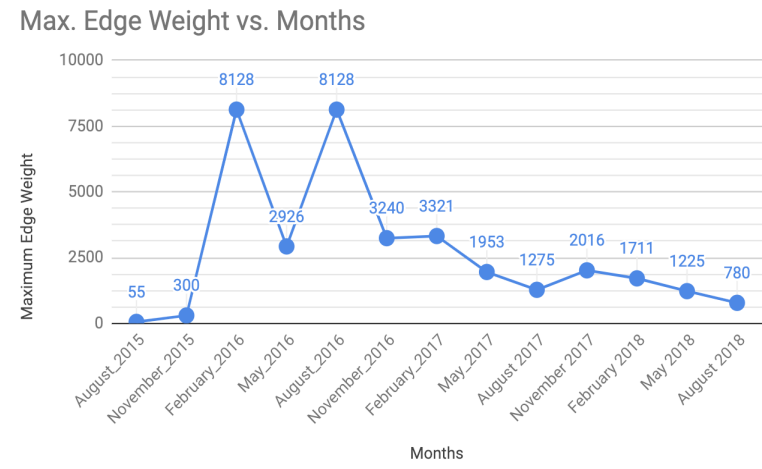

Figure 8: Maximum user edge weights in the User-to-User network model for "The _Donald" user network, spanning from Aug 2015 to Aug 2018. The two spikes indicated in red mark the peak of connectivity during the 2016 US Presidential election.

4.2.1 Total Average Downtime Between Comments. In Figure 9, the two users with the maximum edge weight of 3240 have lower average time between two comments are "TrumpTrain-bot" and "trumpcoatbot". The user with the lowest average time between comments is 3 seconds by "AutoModerator", which is a feature of Reddit that poses as a user who autonomously mod- erates the content. With outliers accounted for, one would expect there is a trend where higher maximum edge weights would indicate a lower average time between comments made within a month.

For edge weights scoring from 0 to 600 , there are users with average time between comments within 2,500 seconds to 11,000 seconds. However for all edge weights, there exists users with under 2,500 seconds as their average time between comments. These results do not present a clear positive correlation between the maximum edge weights and average time between comments. The uncertainty can be reduced by focusing on averaging the time-stamps of bursts of comments rather than averaging time-stamps over a month's period. To this end, we change the month's period to a burst of comments that occur within within one minute.

4.2.2 Burst Average Downtime Between Comments. Figure 10 exhibits the user comment down time within a one minute burst. The results indicate that the majority of users possess a down time of 30 seconds to 45 seconds. The two users with the maximum edge weight 3024 have down times below the average cluster of users, these are "Trumptrain-bot" and "trumpcoatbot". The user with the lowest down time between comments is the "Automoderator" which is expected to have fast down times because of its multithreaded function available on the Reddit platform.

The burst temporal analysis indicates that the maximum edge weight metric stratifies the user dataset where users with higher edge weights tend to rank lower in the average down time between comments.

An extension of our study would to also record the average burst down time between comments in just the "The_Donald" subreddit page. We hypothesize that this would reveal the users focused solely on contributing to "The_Donald" related content and in turn

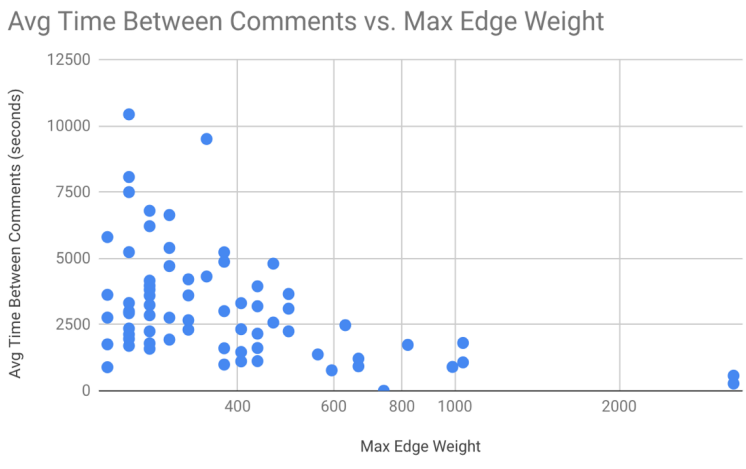

Figure 9: A comparison of the maximum edge weight between two user nodes and their individual user average time between all comments made in seconds by them on Reddit. This data is taken from 68 users within the subreddit “The_Donald” in November 2016.

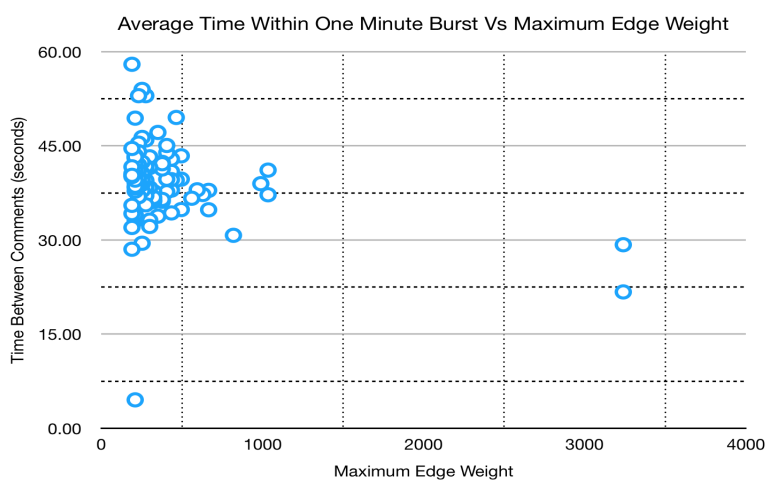

Figure 10: Each data point represents one user in the scope of their average downtime between commenting within one minute burst with respect to their maximum edge weight. Data taken from total Reddit comments in November 2016.

reveal a clearer correlation between maximum edge weights and average down time between comments.

\section{CONCLUSION AND FUTURE WORK}

Because the Reddit platform structure is divided into topic pages and relies on content propagation by users, it proves to be a successful environment to scrutinize an online microcosm. The postto-post network simplified the dataset by extracting the posts that share users. The user-to-user network yielded a method for visualizing the hyper-connectivity between those users.

After testing the network models on three subreddits, we have noted that subreddit "The_Donald" contained inflated maximum edge weights in comparison to the other two equally populated subreddits. The temporal data analysis has revealed a trend between increasing maximum edge weight and decreasing time interval between a user's comments. This inversely proportional relationship suggests that those who rank abnormally high in the 
maximum edge weight metric are, in turn, likely to exhibit abnormally low down time between comments. We can then conclude that the maximum edge weight metric is therefore successful in identifying abnormal users, bots among them, within the Reddit social media platform.

Future work will entail strengthening the edge weight metric by incorporating the Rest-Sleep-and-Comment pattern [9] to verify the anomalous accounts.

\section{ACKNOWLEDGMENTS}

This work was supported in part by CyLab at Carnegie Mellon University under grant NGIT2009100109 from the Northrop Grumman Cybersecurity Research Consortium (NGCRC).

\section{REFERENCES}

[1] Jose Brustoloni, Nicholas Farnan, Ricardo Villamarin-Salomon, and David Kyle 2009. Efficient Detection of Bots in Subscribers' Computers. In 2009 IEEE International Conference on Communications. 1-6.

[2] Chiyu Cai, Linjing Li, and Daniel Zengi. 2017. 2017 IEEE International Conference on Intelligence and Security Informatics (ISI). In Behavior Enhanced Deep Bot Detection in Social Media. 128-130.

[3] Qiang Cao, Michael Sirivianos, Xiaowei Yang, and Tiago Pregueiro. 2012. Aiding the Detection of Fake Accounts in Large Scale Social Online Services. In
Proceedings of the 9th USENIX Conference on Networked Systems Design and Implementation (NSDI'12). USENIX Association, Berkeley, CA, USA, 15-15.

[4] CrowdSource. [n. d.]. Bottiquette. reddit.com/r/Bottiquette/wiki/bottiquette

[5] Clayton Allen Davis, Onur Varol, Emilio Ferrara, Alessandro Flammini, and Filippo Menczer. 2016. BotOrNot. Proceedings of the 25th International Conference Companion on World Wide Web - WWW'16 Companion (2016). https://doi.org/10.1145/2872518.2889302

[6] Ryan Detert. 2018. Bot or Not: Seven Ways To Detect An Online Bot.

[7] Yury Drevs and Aleksei Svodtsev. 2016. Formalization of Criteria for Social Bots Detection Systems. Procedia - Social and Behavioral Sciences 236 (2016), 9 - 13. https://doi.org/10.1016/j.sbspro.2016.12.003 International Conference on Communication in Multicultural Society, CMSC 2015, 6-8 December 2015, Moscow, Russian Federation.

[8] Emilio Ferrara, Onur Varol, Clayton Davis, Filippo Menczer, and Alessandro Flammini. 2016. The rise of social bots. Commun. ACM 59, 7 (Jun 2016), 96104. https://doi.org/10.1145/2818717

[9] Alceu Ferraz Costa, Yuto Yamaguchi, Agma Juci Machado Traina, Caetano Traina, and Christos Faloutsos. 2015. RSC. Proceedings of the 21th ACM SIGKDD International Conference on Knowledge Discovery and Data Mining - KDD '15 (2015). https://doi.org/10.1145/2783258.2783294

[10] Sharath Chandra Guntuku, Pratik Narang, and Chittaranjan Hota. 2013. Realtime Peer-to-Peer Botnet Detection Framework based on Bayesian Regularized Neural Network. (07 2013). arXiv:1307.7464 https://arxiv.org/pdf/1307.7464

[11] Kiel Long, John Vines, Selina Sutton, Phillip Brooker, Tom Feltwell, Ben Kirman, Julie Barnett, and Shaun Lawson. 2017. "Could You Define That in Bot Terms"? Proceedings of the 2017 CHI Conference on Human Factors in Computing Systems - CHI '17 (2017). https://doi.org/10.1145/3025453.3025830

[12] Office of the Director of National Intelligence. 2017. Assessing Russian Activities and Intentions in Recent US Election. Intelligence Community Assessment. 